\title{
UREAD Impact Behaviour Using Silicon Based Materials
}

\author{
Remi Bouttier ${ }^{1}$, Gabriel Lopes ${ }^{2}$, Luke Clarke ${ }^{3}$, Rocco Lupoi ${ }^{3, ~ *}$ \\ ${ }^{1}$ Ecole Nationale Superieure de Mechanique et D’Aerotechnique (ISAE-ENSMA), Département d'Energétique, France \\ ${ }^{2}$ Federal University of Uberlandia, Engenharia Mecânica, Santa Mônica, Uberlândia - MG, Brazil \\ ${ }^{3}$ Trinity College Dublin, the University of Dublin, Department of Mechanical and Manufacturing Engineering, Parsons Building, Dublin 2, \\ Ireland
}

\section{Email address:}

lupoir@tcd.ie (R. Lupoi)

\section{To cite this article:}

Remi Bouttier, Gabriel Lopes, Luke Clarke, Rocco Lupoi. UREAD Impact Behaviour Using Non-Newtonian Materials. International Journal of Mechanical Engineering and Applications. Vol. 3, No. 4, 2015, pp. 57-62. doi: 10.11648/j.ijmea.20150304.12

\begin{abstract}
Several methodologies and techniques are currently available so as to dissipate energy in engineering systems; most of them are either not re-usable, or complex in mechanism. This paper introduces an innovative re-usable energy absorption device, based upon the working principles of Equal Channel Angular Extrusion, and known as UREAD (Universal Re-usable Energy Absorption Device). This study compares the behaviour of different "low-density" deformable materials (a range of silicon rubber grades) inserted in a UREAD unit and loaded under impact condition. The energy absorbed was experimentally measured and compared against the impact energy. It was possible to dissipate levels as high as $74.91 \%$ of the impact energy when using a simple set-up, and the device re-usability was demonstrated.
\end{abstract}

Keywords: ECAE, Energy Absorption, UREAD, Non-Newtonian Materials, Impact

\section{Introduction}

Impacts, from road accidents to earthquakes, are threats to humans and their belongings. The scale of car, airplane and ship collisions are different than the collisions that occur naturally like in earthquakes [1]. Several technologies and methods were devised in order to prevent or minimize the damages due to these events, however the implementation of a final solution with maximum efficiency is yet far from being achieved. The development of current technologies and the engineering of new methods is currently driving the work of scientists in the field.

There are different types of energy absorbing devises based on a set number of working principles. Several methods have been used to design new and innovative systems, and a considerable research work has so far been carried out so as to find efficient ways to dissipate the energy from an impact. Materials in not traditional forms have been studied for their ability to absorb impacts, such metallic foams. Different types of foams have been explored, i.e. aluminium [2,3], and Ni/Al-hybrid [4]. It has been demonstrated possible that the engineering of objects with specific shapes can increase energy dissipation capabilities, this is the case for honeycomb and thin-wall structures. Their characteristics have been extensively explored, with interesting applications. As an example, experimental and numerical studies of honeycomb structures were made to unravel its crashworthiness parameters [5], and its absorption performance when applied to motorbike helmets [6]. However, honeycomb structures can be rather complex to manufacture and are not efficient if the impact force forms a small angle with the honeycomb cells plane. Thin-walled structures has also been explored as energy absorbers [7,8], but their typical performance under radial compression is poor. Besides that, they can only dissipate energy when they buckle, and once a first loading has occurred the unit is not re-usable. Car bumpers are also examples of energy dissipation devices based upon plastic deformation, not deemed re-usable. A design and FEA crash simulation for a composite car bumper is reported in [10], an optimized bumper systems for pedestrian lower-leg protection in [11], and an experimental study on characteristics of shock absorbers of impact energy of passenger coaches in [12]. This is also the case for a new generation of guardrails [9]; like with the others the nature of the working mechanism will not allow re-usage.

Such non-reusability characteristic is typical for "passive" shock absorbers, but a number of re-usable systems exist, and are deemed as "active". This is the case for a linear permanent-magnet motor for active vehicle suspension [13]. Examples are a permanent magnet eddy current brake for a 
small scaled electromagnetic launch model [14], and a system to stop roller coasters by using magnets on the train to induce eddy currents in the braking fins [15]. Other examples of active systems are advanced types of friction dampers, which can be used over a wide range of applications, i.e. for active support of piezoelectric ceramic actuators [16]. Frictional based dampers in earthquake resistant structures is another application, that has advantages over other types of energy dissipating devices. These include low cost of manufacturing and maintenance and also being less susceptible to environmental effects. Furthermore, no yielding occurs in such dampers after a severe earthquake which eliminates the need for replacement [17]. Semi-active system with friction dampers for lightweight pedestrian bridges is another example [18]. An alternative is represented by an energy-harvesting shock absorber with a mechanical motion rectifier [19]. To summarize, active or semi-active systems can consume a significant amount of energy to be efficient at impact. They are still rather complex and are currently implemented only at large o very large scale.

This paper presents experimental results obtained by using a novel passive energy absorber, known as UREAD, based upon the principles of materials plastic deformation but in such a way to allow for re-usability. It has the potential to overcome to some disadvantages of current systems. The device has been initially explored and results published in $[20,21]$, however primarily with Lead as deformable material. This paper will expand to the testing of alternative lighter-weight and not metallic materials.

\section{Experiments set up}

\subsection{UREAD Working Mechanisms and Test Unit}

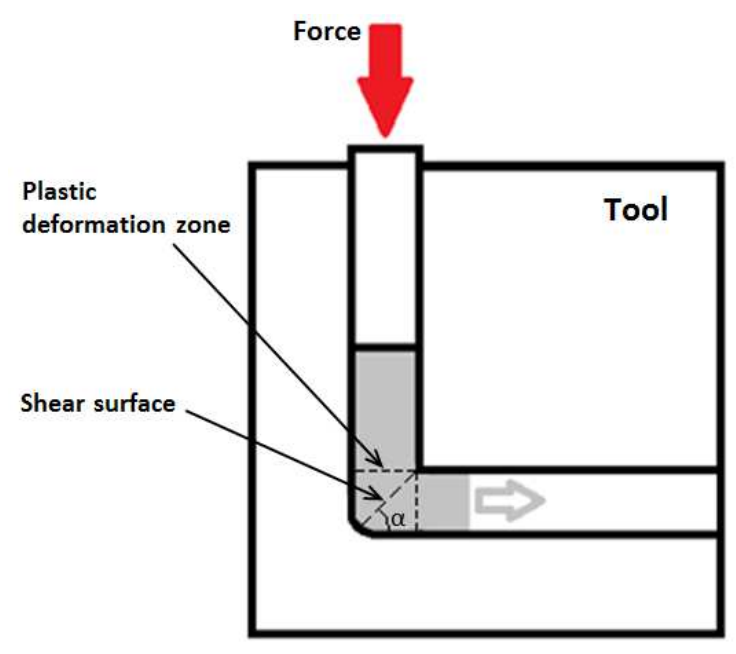

Figure 1. Shearing process of materials in $90^{\circ}$ intersecting channels.

The Universal Reusable Energy Absorption Device (UREAD) working mechanism is based upon the principles Equal Channel Angular Extrusion (ECAE), and it is shown in Figure 1. A material is pushed through a channel of defined cross-section, with a change of direction of a determined angle. The device can be designed with different cross- sections types, such as circular or hexagonal; while the deformation shape can be different such as with U-channels [22].

When an external force is applied to the pushing punch, the solid billet will be forced to change its moving direction at correspondence of the channel bends, in order to follow the geometry of the channel; hence energy will be dissipated by a plastic deformation shearing process [22]. While most of plastic deformations are irreversible, the fact that this one occurs inside a closed channel makes it controllable and then reversible: it only needs a push from the other side to come back to its original geometry.

This process can be explained by the diagram in Figure 1, which describes the plastic deformations mechanism occurring on a material when being moved within two intersecting channels. In order to achieve the material motion, shear has to happen at the intersection zone. As Figure 2 shows, the shearing process is modelled to develop on a shear surface, inclined at an angle $\alpha$. The plastic deformation model for this process is based upon the principles of the Upper Bound Analysis technique [23]. It is clear that for this design, the material can be pushed forward and backwards through the two channels, potentially for an infinite number o times.

Figure 2(a) shows the UREAD unit which was used for the experimental work presented in this article. It is made out of tool steel (both unit and pressing punch), and has 2 channel cross-sectional geometries, however both rectangular, intersecting at $90^{\circ}$ degrees, i.e. $8 \times 8 \mathrm{~mm}$ and $10 \times 10 \mathrm{~mm}$. For the purpose of this study only the $10 \times 10 \mathrm{~mm}$ channel was used. The billet of deformable material is visible from the figure, positioned in this case in the $10 \times 10 \mathrm{~mm}$ channel. In this design the channels are covered with a flat steel plate so as to build a fully assembled UREAD, as shown in Figure 2(b). (a)

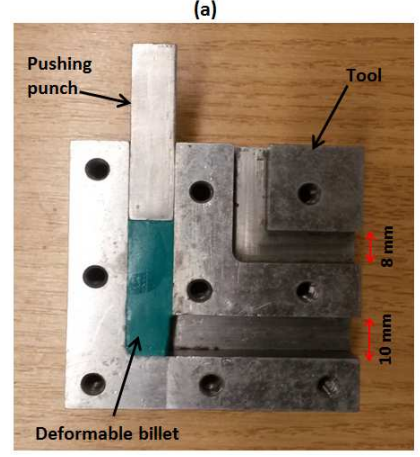

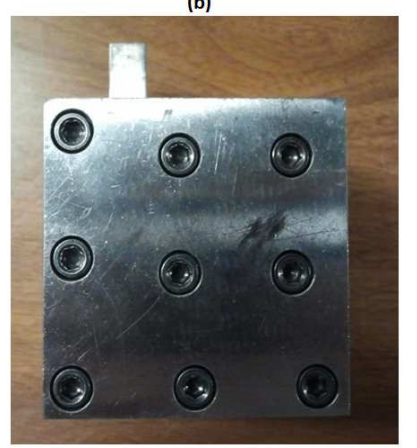

Figure 2. (a) UREAD unit with square cross-sectional channels. (b) UREAD cover plate used in this set-up.

\subsection{Deformable Materials and Impact Test}

In order to test the UREAD unit under impact conditions, an experimental apparatus was developed to resemble a hammer-drop test rig where the impact force is measured with a force sensor. Figure 3 shows an overview image of the set up. It consists of a hollow steel tube $965 \mathrm{~mm}$ long; a weight is inserted in the top side of the tube, and made to 
impact on a UREAD unit located just above the ground. Figure 4 shows a close-up picture of the UREAD zone. It appears clear from the image the unit is bolted to a device support plate, and that the force sensor is firmly inserted between the device support plate and ground plate. The sensor used is an Integrated Circuit Piezoelectric (ICP) type purchased from PCB Piezotronics Inc. (model 208C05). A transducer is also used (signal conditioner purchased from PCB Piezotronics Inc., model 480C02). The data acquisition software is Labview.

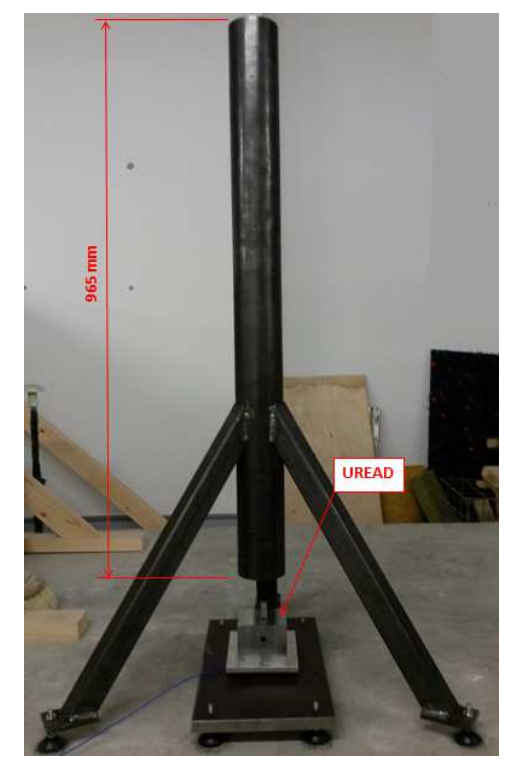

Figure 3. Impact test rig.

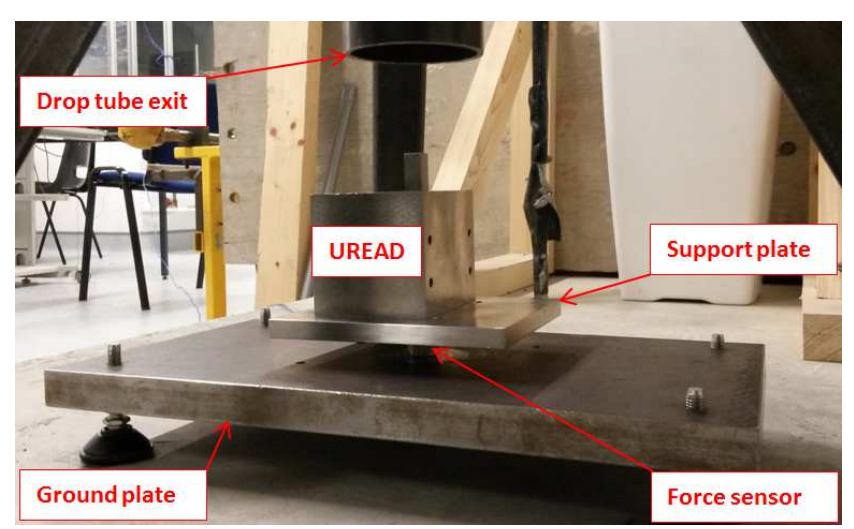

Figure 4. Close-up image of impact location.

For all impact tests a billet of deformable material measuring $30 \mathrm{~mm}$ in height was shaped to fit the $10 \times 10 \mathrm{~mm}$ channel geometry, in a similar manner as it appears in Figure 1(a).

The deformable materials used are soft-dilatant polymers (silicon rubber), purchased from SportsHealth and sold under the commercial name of "Power Putty". Four commercial grades were tested known as Soft, Medium-Soft, MediumFirm and Firm. A sample of each grade was characterized through a quasi-static compression test; a typical elastoplastic behaviour was observed in all cases. It was possible to identify the Yield Stress and Young Modulus (E), which have been reported in Table 1 for all the grades used in the experiments. It is shown the material mechanical characteristics enhance from Soft to Firm.

Table 1. Deformable materials characteristics.

\begin{tabular}{lll}
\hline Material & Yield Stress [Pa] & Young Modulus [MPa] \\
\hline Soft & 70 & 0,024 \\
Medium Soft & 90 & 0,042 \\
Medium Firm & 270 & 0,21 \\
Firm & 850 & 0,46 \\
\hline
\end{tabular}

Following the billet preparation, the pressing punch in UREAD was placed in its location. The device is then closed and tightened with nine screws to a steel plate of $10 \mathrm{~mm}$ thickness, as already described in Figure 2(b).

It is necessary for the drop tube to be aligned with the UREAD unit pressing punch on an axial direction, so as to make sure the weight impacts on the punch along its axis. This is to avoid the generation of bending moments, the formation of which would not be possible to take into account with the available experimental set-up. This was done, and the relative location of the UREAD unit marked so that it could be re-positioned after each test.

In order to generate a range of impact energies, 3 weights with different mass and drop heights are used. The weights are cylindrical to fit in the dropping tube and they are connected to a hook, which is used to hold each weight before being dropped. Table 2 shows the used weights masses and heights of fall, alongside with additional relevant data.

Table 2. Impact test parameters.

\begin{tabular}{llll}
\hline & Total mass [kg] & Weight length [m] & Height of fall [m] \\
\hline Weight 1 & 1.134 & 0.040 & 0.994 \\
Weight 2 & 1.689 & 0.060 & 0.984 \\
Weight 3 & 2.265 & 0.080 & 0.974 \\
\hline
\end{tabular}

\section{Results and discussion}

To calculate the total energy at the instant of impact for each weight the simple kinetic energy formula was used, as in Equation 1. Thus, Equation 1 and Equation 2 were used so as to calculate impact energy and velocity.

$$
\begin{gathered}
E_{\text {impact }}=(1 / 2) m v^{2} \\
v^{2}=v_{o}{ }^{2}+2 g \Delta h
\end{gathered}
$$

Where $m$ is the mass of the weight, $v$ is the velocity of the weight just before the impact on the punch, $v_{o}$ is initial velocity of the weight (which equals to zero), $g$ is the acceleration due to gravity and $\Delta h$ is the height of fall.

The values obtained are shown in Table 3:

Table 3. Generated impact energies.

\begin{tabular}{ll}
\hline & Total Impact Energy [J] \\
\hline Weight 1 & 11.05 \\
Weight 2 & 16.29 \\
Weight 3 & 21.63 \\
\hline
\end{tabular}


On the other hand, Equation 3 is used to calculate the amount of energy, $E_{U R E A D}$, the UREAD device was able to absorb. It is therefore not assumed the full impact levels by Equation 1 are absorbed by the unit on its own.

$$
E_{U R E A D}=F_{\text {ave }} \cdot s
$$

Where $F_{a v e}$ is the average force during the impact (by the force sensor), and $s$ is the total punch displacement, i.e. the distance the punch has travelled (measured after the impact has occurred).

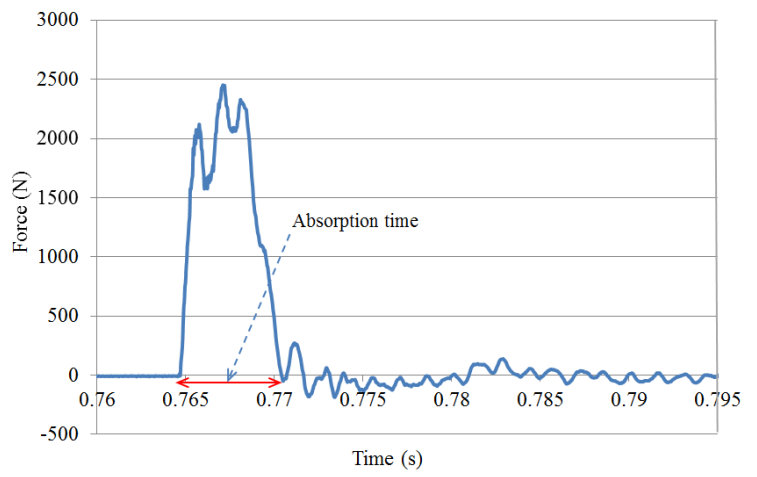

Figure 5. UREAD measured force vs time during an impact using the Firm grade.

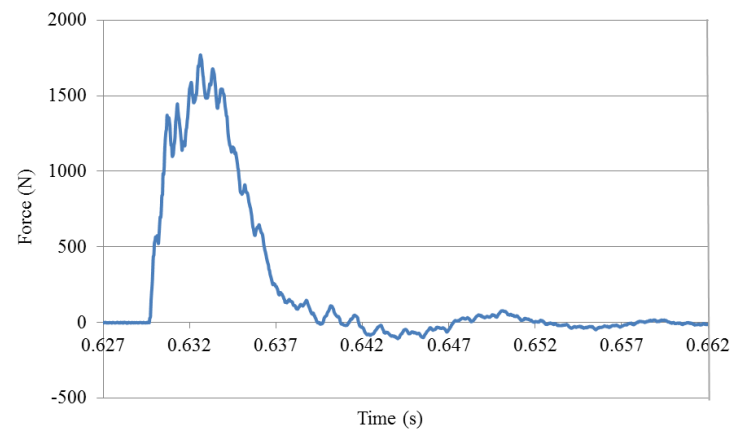

Figure 6. UREAD measured force vs time during an impact using the Soft grade.

Figure 5 and Figure 6 show examples of force measurements over time during an impact, where $E_{\text {impact }}$ is the same in both cases. The data acquisition frequency was set to $30 \mathrm{kHz}$. Specifically, Figure 5 is for an impact using the Firm grade in the UREAD channel, loaded with Weight 2; while Figure 6 is for an impact with the Soft grade, also using Weight 2 by following the fall parameters in Table 2. The average force, $F_{\text {ave }}$, has been calculated over the absorption time (highlighted in Fugure 5). Such corresponds to the period of time the channel punch is actually moving, hence energy is absorbed by the UREAD unit. In both cases the force characteristic rapidly rises as the weight lands on the channel punch, up to the reach of a peak value. This value is higher for the Firm grade, result in agreement with the material characteristics reported in Table 1. On the other hand the absorption time is longer, nearly double, for the Soft grade. This means in the latter case the punch has travelled a longer distance, despite $E_{\text {impact }}$ has been kept constant. A residual vibrational mode was observed for periods of time beyond the actual impact, also visible in Figure 5 and Figure 6; this was attributed to a post-impact effect to the UREAD and load cell mounting/holding structures.

In the two experiments the recorded peak force was in the order of $2.5 \mathrm{kN}$ for the Firm grade, and $1.7 \mathrm{kN}$ for the Soft grade. An additional test was executed using the same impact conditions, however no deformable material was inserted in the channel, i.e. the UREAD unit was not active. In this case a peak impact force of $14.5 \mathrm{kN}$ was recorded, approximately 6 times higher as compared to when UREAD is functional. This clearly demonstrates it was possible using UREAD to dissipate energy and cut peak force levels considerably in the experiments. Such is a critical feature for an energy absorber.

The experimental results using the impact energies as by Table 3, and with the combination of the considered material grades, are summarized in Table 4. An average was calculated and reported in the table. The energy absorbed was estimated using Equation 3, while the frequency of data acquisition was $30 \mathrm{kHz}$ in all cases. Such is the maximum value as by the force sensor specifications. All experiments were repeated, giving comparable results.

Table 4. Summary table of impact tests results.

\begin{tabular}{|c|c|c|c|c|c|}
\hline & $\begin{array}{l}\text { Impact Average Force } \\
{[\mathrm{N}]}\end{array}$ & $\begin{array}{l}\text { AbsorptionTime } \\
{[\mathrm{ms}]}\end{array}$ & $\begin{array}{l}\text { Final Punch Displacement } \\
{[\mathrm{mm}]}\end{array}$ & $\begin{array}{l}\text { Energy Absorbed } \\
{[\mathrm{J}]}\end{array}$ & $\begin{array}{l}\text { Percentage of Energy Absorbed } \\
{[\%]}\end{array}$ \\
\hline \multicolumn{6}{|c|}{ WEIGHT 1 - 1.134 KG (IMPACT ENERGY = $11.05 \mathrm{~J}$ ) } \\
\hline Very Soft & 865.63 & 6.17 & 7.33 & 6.36 & 57.55 \\
\hline Soft Medium & 1061.18 & 5.59 & 4.94 & 5.31 & 48.04 \\
\hline Medium Firm & 1145.94 & 5.15 & 4.57 & 5.23 & 47.31 \\
\hline Firm & 1313.68 & 4.74 & 3.37 & 4.42 & 39.96 \\
\hline \multicolumn{6}{|c|}{ WEIGHT 2 - $1.689 \mathrm{KG}$ (IMPACT ENERGY = 16.29 J) } \\
\hline Very Soft & 822.32 & 9.37 & 12.05 & 9.96 & 61.09 \\
\hline Soft Medium & 989.74 & 8.16 & 9.92 & 9.81 & 60.18 \\
\hline Medium Firm & 1302.64 & 6.44 & 7.55 & 9.76 & 59.91 \\
\hline Firm & 1533.89 & 5.62 & 6.43 & 9.87 & 60.54 \\
\hline \multicolumn{6}{|c|}{ WEIGHT 3 - 2.265 KG (IMPACT ENERGY = 21.63 J) } \\
\hline Very Soft & 816.25 & 12.40 & 16.60 & 13.59 & 62.80 \\
\hline Soft Medium & 1232.66 & 7.75 & 13.23 & 16.21 & 74.91 \\
\hline Medium Firm & 1385.62 & 7.18 & 11.38 & 15.78 & 72.92 \\
\hline Firm & 1403.05 & 7.64 & 9.78 & 13.71 & 63.38 \\
\hline
\end{tabular}


Figure 7 to Figure 9 show a graph of the absorbed energy, $E_{U R E A D}$, for each material grade (from Soft to Firm) using the three levels of impact energy, $E_{\text {impact }}$, obtained with Weight 1 , 2 and 3.

When the impact energy is the lowest (Figure 7), the Soft grade performs the best and absorbs $57.55 \%$ of the impact. The Firm grade, on the other hand, does not cross the $40 \%$ level. As the impact energy increases (Figure 8 and Figure 9), the dissipation performance trend becomes more uniform. With Weight 3 all of the grades performed very similarly by absorbing approximately $70 \%$ of the impact, with a peak of $74.91 \%$ using the Soft Medium. This behaviour emphasizes that the choice of deformable material is very critical, and careful considerations must be made in order to select the grade to suits best the application needs. Experimental results also demonstrate the versatility of UREAD is very high; it was in fact possible to dissipate a range of energy levels by interchanging the deformable material only, while the overall geometry remained unchanged. At the same time, the employment of lighter-weight materials in UREAD channels has been proved to perform efficiently, and it can be considered as a valid alternative to Lead or other metals as deformable billet so far being used in the UREAD technology.

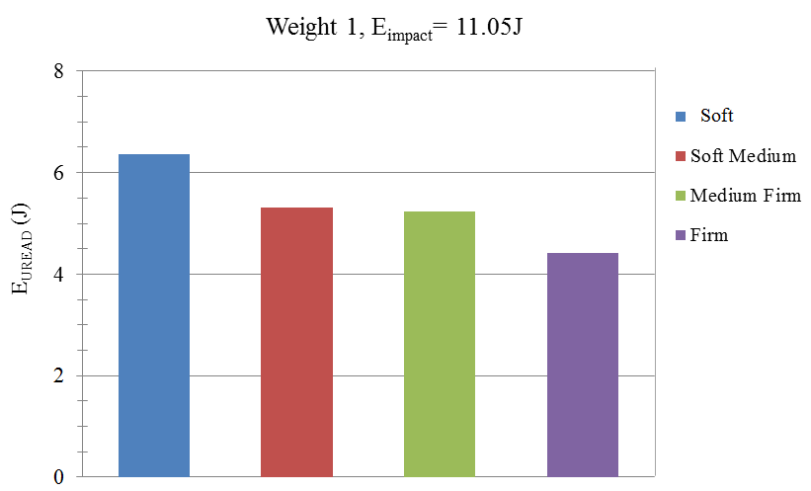

Figure 7. Comparison of the absorbed energy for the material grades with weight 1 .

Weight 2, $\mathrm{E}_{\text {impact }}=16.29 \mathrm{~J}$

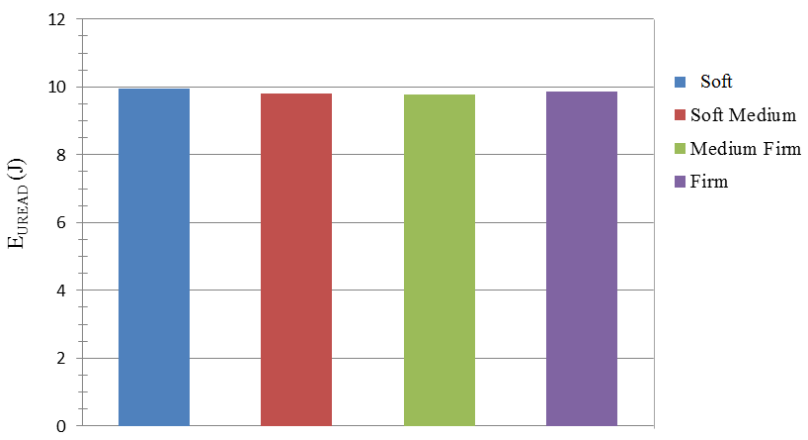

Figure 8. Comparison of the absorbed energy for the material grades with weight 2 .

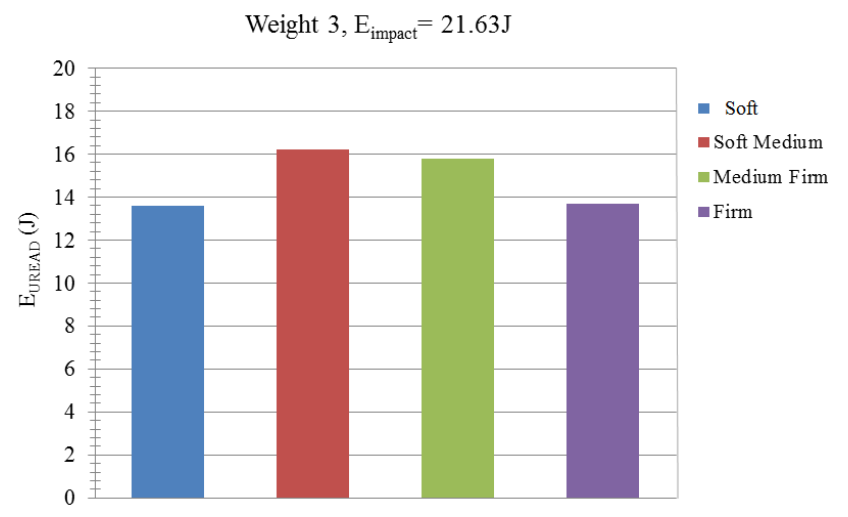

Figure 9. Comparison of the absorbed energy for the material grades with weight 3

Figure 10 summarises the specific behaviour for each material. The Soft grade is the most effective for low impact energies (up to just above 15J), however the effectiveness does not vary much when the actual impact energy increases (quasi-linear curve). The effectiveness of the "medium" grades (Soft Medium and Medium Firm) is rather similar, with a significant increase after approximatively $11.5 \mathrm{~J}$. The Firm material grade is the less effective for lower impact energies; it is however forecasted to be more suitable for higher energy tests which were not considered for the scope of this work..

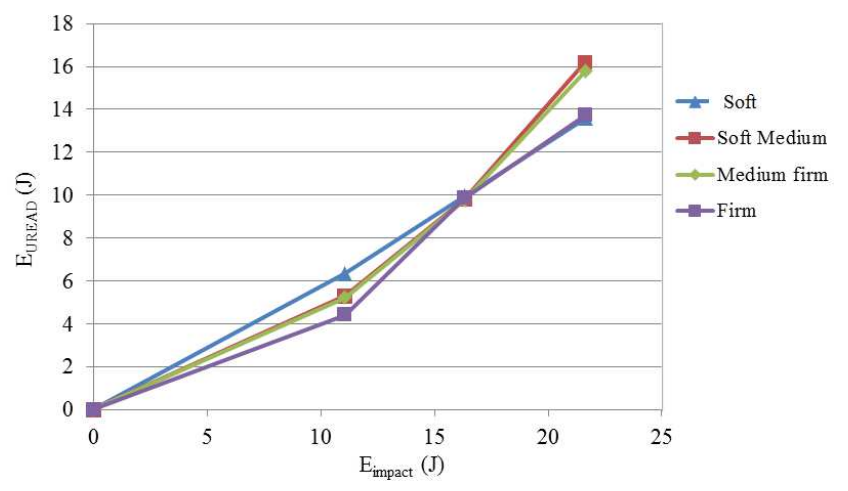

Figure 10. Evolution of the absorbed energy vs impact energy.

\section{Conclusions}

A novel technology, known as UREAD, capable of dissipating energy in engineering systems was introduced. It is based upon principles of Equal Channel Angular Extrusion (ECAE), with the ability of being re-usable after loading. This is not a typical characteristic of passive energy absorbers.

Impact tests were carried out on a UREAD device using silicon based materials as being deformed in the channel. Four different grades were tested, covering a yield stress range from $70 \mathrm{~Pa}$ up to $850 \mathrm{~Pa}$ measured under static compression.

A in-house drop-hammer rig was used in order to simulate different levels of impact energy. The average force during 
the impact period was used to calculate the energy absorbed by the device and it was compared to the impact energy. For lower impact energies $(<15 \mathrm{~J})$, the softer Soft grade is the most efficient energy absorber as it dissipated $57.55 \%$ of it. However, for higher impact energies (between $16 \mathrm{~J}$ and 21J), the Medium Firm and Medium Soft were shown to be able to dissipate up to $72.92 \%$ and $74.91 \%$ of the impact respectively. It was therefore possible to demonstrate the high versatility level of UREAD, as a variety of energy level was dissipated while the overall geometry remained unchanged. After each test it was possible to re-use the same UREAD unit and deformable material.

In general, all grades were able to dissipate increasing values of energy as the impact levels also increased. It is concluded such new materials represent a valid alternative and a lighter weight option to metals in UREAD applications.

\section{Acknowledgements}

The authors would like to acknowledge the support of the ERASMUS and Science Without Borders (SWB - funded by CAPES, Brazil) schemes for enabling this project. The authors are also thanking Prof. Ciaran Simms (TCD) for the support in the execution of the work presented in this article.

\section{References}

[1] Alghamdi AAA. Collapsible impact energy absorbers: An overview. Thin-Walled Structures 2001; 39:189-213.

[2] Rajendran R, Prem Sai K, Chandrasekar B, Gokhale A, Basu S. Preliminary investigation of aluminium foam as an energy absorber for nuclear transportation cask. Materials and Design 2008; 29:1732-9.

[3] Kim A, Hasan MA, Nahm SH, Cho SS. Evaluation of compressive mechanical properties of Al-foam using electrical conductivity. Composite Structures 2005; 71:191-8.

[4] Jung A, Lach E, Diebels S. New hybrid foam materials for impact protection. International Journal of Impact Engineering $2014 ; 64: 30-8$.

[5] Partovi Meran A, Toprak T, Muğan A. Numerical and experimental study of crashworthiness parameters of honeycomb structures. Thin-Walled Structures 2014; 78:87-94

[6] Caserta GD, Iannucci L, Galvanetto U. Shock absorption performance of a motorbike helmet with honeycomb reinforced liner. Composite Structures 2011; 93:2748-59.

[7] Abramowicz W. Thin-walled structures as impact energy absorbers. Thin-Walled Structures 2003; 41:91-107.

[8] Yang Z, Yan H, Huang C, Diao X, Wu X, Wang S, Lu L, Liao L, Wei Y. Experimental and numerical study of circular, stainless thin tube energy absorber under axial impact by a control rod. Thin-Walled Structures 2014; 82:24-32.

[9] Atahan AO, Yücel AÖ, Erdem MM. Crash testing and evaluation of a new generation L1 containment level guardrail. Engineering Failure Analysis 2014; 38:25-37.

[10] Calienciug A, RADU GhN. Design and FEA crash simulation for a composite car bumper. Bulletin of the Transilvania University of Brasov, Series I: Engineering Sciences 2012; 5(1):7-12.

[11] Jiang K, Yang J. Optimization of bumper system for pedestrian lower leg protection from vehicle impact. Third International Conference on Digital Manufacturing \& Automation. 2012.

[12] Simić G, Lučanin V, Tanasković J, Radović N. Experimental research of characteristics of shock absorbers of impact energy of passenger coaches. Experimental Techniques 2009; 29-35.

[13] Wang J, Wang W, Atallah K. A linear permanent-magnet motor for active vehicle suspension. IEEE Transactions On Vehicular Technology 2011; 60(1):55-63.

[14] Zhou S, Yu H, Hu M, Huang L. Design of permanent magnet eddy current brake for a small scaled electromagnetic launch model. Journal of Applied Physics III 2012; 07A738:1-3.

[15] Pendrill AM, Karlsteen M, Rödjegård H. Stopping a roller coaster train. Physics Education 2012; 47(6):728-35.

[16] Mingfu L, Mingbo S, Siji W. Active Elastic Support/Dry Friction Damper with Piezoelectric Ceramic Actuator. Shock and Vibration 2014; ID 712426:1-10.

[17] Samani HR, Mirtaheri M, Zandi AP, Bahai H. The Effects of Dynamic Loading on Hysteretic Behavior of Frictional Dampers. Shock and Vibration 2014; ID 181534:1-9.

[18] Wieczorek N, Gerasch WJ, Rolfes R, Kammerer H. Semiactive Friction Damper for Lightweight Pedestrian Bridges. Journal of Structural Engineering 2014; 140:1-13.

[19] Li Z, Zuo L, Kuang J, Luhrs G. Energy-harvesting shock absorber with a mechanical motion rectifier. Smart Materials and Structures 2013; 22:1-10.

[20] Lupoi R, Osman FH. Loading behaviour of 900 UREAD energy channels. International Journal of Crashworthiness 2008, 13, 2: 195-203.

[21] Osman F.H., Lupoi R. Application of "UREAD" for the energy dissipation in engineering structures. Key-Engineering Materials, 486, 2011, 1-4.

[22] Lupoi R. Investigation into Energy Dissipation in Equal Channel Angular Extrusion. PhD Thesis. University of Bath (UK), Department of Mechanical Engineering, 2008.

[23] Hosford W.F., Caddell R.M. Metal Forming: Mechanics and Metallurgy. Cambridge University Press, 4th edition, 2014. 